\title{
Cross-Species Transmission and Evolution of SIV Chimpanzee Progenitor Viruses Toward HIV-1 in Humanized Mice
}

\author{
Kimberly Schmitt ${ }^{1+}$, James Curlin ${ }^{1 \dagger}$, Leila Remling-Mulder ${ }^{1}$, Ryan Moriarty ${ }^{2}$, Kelly Goff ${ }^{3}$, \\ Shelby O'Connor', Mark Stenglein ${ }^{1}$, Preston Marx ${ }^{3,4}$ and Ramesh Akkina ${ }^{1 *}$ \\ 1 Department of Microbiology, Immunology, and Pathology, Colorado State University, Fort Collins, CO, United States, \\ ${ }^{2}$ Department of Pathology and Laboratory Medicine, School of Medicine and Public Health, University of Wisconsin, \\ Madison, WI, United States, ${ }^{3}$ Tulane National Primate Research Center, Tulane University, Covington, LA, United States, \\ ${ }^{4}$ Department of Tropical Medicine, School of Public Health \& Tropical Medicine, Tulane University, New Orleans, LA, \\ United States
}

OPEN ACCESS

Edited by:

Fatah Kashanchi,

George Mason University,

United States

Reviewed by:

Tetsuro Matano,

National Institute of Infectious

Diseases (NIID), Japan

Rajesh Thippeshappa,

Texas Biomedical Research Institute,

United States

${ }^{*}$ Correspondence:

Ramesh Akkina

akkina@colostate.edu

${ }^{\dagger}$ These authors share first authorship

Specialty section:

This article was submitted to Virology,

a section of the journal

Frontiers in Microbiology

Received: 22 May 2020

Accepted: 20 July 2020

Published: 11 August 2020

Citation:

Schmitt K, Curlin J,

Remling-Mulder L, Moriarty R, Goff K, O'Connor S, Stenglein M, Marx P and Akkina R (2020) Cross-Species Transmission and Evolution of SIV Chimpanzee Progenitor Viruses

Toward HIV-1 in Humanized Mice.

Front. Microbiol. 11:1889.

doi: 10.3389/fmicb.2020.01889
The genetic evolution of HIV-1 from its progenitor virus SIV following cross-species transmission is not well understood. Here we simulated the SIVcpz initial transmission to humans using humanized mice and followed the viral evolution during serial passages lasting more than a year. All three SIVcpz progenitor viruses used, namely LB715 and MB897 (group M) as well as EK505 (group N) readily infected hu-mice resulting in chronic viremia. Viral loads increased progressively to higher set-points and the CD4 ${ }^{+}$ $T$ cell decline became more pronounced by the end of the second serial passage indicating viral adaptation and increased pathogenicity. Viral genomes sequenced at different time points revealed many non-synonymous variants not previously reported that occurred throughout the viral genome, including the gag, pol, env, and nef genes. These results shed light on the potential changes that the SIVcpz genome had undergone during the initial stages of human infection and subsequent spread.

Keywords: HIV-1 evolution from SIVcpz, cross-species viral transmission, SIVcpz evolution into HIV-1, modeling SIV evolution into HIV using humanized mice, SIV pathogenesis in humanized mice, origin of human pathogens in NHP, SIV genetic changes toward HIV-1, viral adaptive changes and genetic evolution

\section{INTRODUCTION}

Throughout history, cross-species transmission of animal viruses to humans gave rise to a number of deadly pathogens including HIV-1. Previous studies indicated that HIV-1 and HIV-2 likely arose from simian immunodeficiency viruses (SIVs) native to chimpanzees (SIVcpz), gorillas (SIVgor), and sooty mangabeys (SIVsm); respectively, into humans (Hirsch et al., 1989; Huet et al., 1990; Sharp et al., 1994; Chen et al., 1996; Hahn et al., 2000; Marx et al., 2001; Keele et al., 2006; Van Heuverswyn et al., 2006; Worobey et al., 2010; Sharp and Hahn, 2011). The chimpanzee-derived viruses are believed to have evolved into the most pathogenic HIV-1 group M viruses and to the less widespread HIV-1 group N, while the gorilla-derived viruses (SIVgor) are believed to have given rise to the rare HIV-1 group O (Keele et al., 2006; Van Heuverswyn et al., 2006; Sharp and Hahn, 2011; Hemelaar, 2012). Numerous other SIVs also exist in a wide range of non-human primates with no evidence of sustained transmission to humans despite frequent contact between 
these primates and humans (Worobey et al., 2010; Sharp and Hahn, 2011). While extensive analysis of HIV-1 and SIVcpz sequences cataloged the differences and similarities between these viruses, the exact mechanism by which the SIV genomes sequentially evolved into the current HIVs capable of efficient replication and causing disease remains to be elucidated. While humans and chimpanzees are highly related from an evolutionary and genetic standpoint, they do harbor very divergent and often unique host restriction factors that provide protection from various viral infections including those from NHP lentiviruses (Wain et al., 2007; Sauter and Kirchhoff, 2019). These factors, in combination with differences in host cell receptors such as $\mathrm{CD}^{+}$and chemokine receptors, such as CCR5 and CXCR4, pose considerable barriers that SIVs need to overcome to evolve into HIV-1 (Hvilsom et al., 2008; Locatelli and Peeters, 2012). A suitable model system needs to be employed to evaluate the critical questions surrounding the initial events of cross-species transmission and viral evolution during serial human propagation.

The new generation of humanized mice harboring a transplanted functional human immune system provides a suitable in vivo human surrogate system to address many questions in the context of SIV evolution (Akkina, 2013). Of the two common models that are currently used, the hu-HSC model is made by injecting human $\mathrm{CD}^{+}{ }^{+}$hematopoietic stem cells (HSC) intrahepatically into the neonatal immunodeficient mice such as Rag2 DK mice, whereas the bone marrow/liver/thymus (BLT) mice are made by surgical transplantation of human fetal liver and thymic fragments under the murine kidney capsule (Garcia and Freitas, 2012; Shultz et al., 2012; Akkina, 2013). These mice produce $\mathrm{T}$ cells, $\mathrm{B}$ cells, macrophages and dendritic cells de novo on a continuous basis. The broad spectrum of these human immune cells at various stages of development makes the hu-mouse model particularly attractive to study human immune cell-tropic viruses (Berges et al., 2010; Denton and Garcia, 2011; Akkina, 2013).

Humanized mice have been used extensively for a host of HIV studies that encompass pathogenesis, sexual transmission and prevention, viral latency and testing of novel therapeutics to name a few (Neff et al., 2010; Denton and Garcia, 2011; Choudhary et al., 2012; Garcia and Freitas, 2012; Charlins et al., 2017; Hu et al., 2017). We and others have recently shown that these mice are permissive for SIV infections as well (Yuan et al., 2016; Schmitt et al., 2017, 2018; Sato et al., 2018; Yuan et al., 2018; Curlin et al., 2020). SIV propagation in hu-mice provides the selective environmental pressures necessary for the virus to potentially overcome human cell-imposed barriers potentially reflecting the original evolution of HIV-1 from its progenitors. Thus, many aspects of cross-species transmission by SIVs into the human host can be experimentally studied. In light of these advantages, recent studies, including ours, addressed the evolution of both HIV-1 and HIV-2 from their SIV ancestor viruses using the hu-mice system (Yuan et al., 2016; Schmitt et al., 2017, 2018; Sato et al., 2018; Yuan et al., 2018; Curlin et al., 2020). With regard to the HIV-2 progenitor virus, we have shown that SIVsm (SIVsmE041) native to sooty mangabeys was able to infect hu-mice and accumulate mutations that increase its fitness to human cells during sequential passages in vivo (Schmitt et al., 2017, 2018). Similarly, recent studies involving SIVcpz, the ancestor of HIV-1, showed that a number of SIVcpz strains can productively infect hu-mice resulting in viremia (Yuan et al., 2016, 2018; Sato et al., 2018; Curlin et al., 2020). Virus isolated after a single passage of 14-16 weeks duration harbored two notable mutations in the env gene (Yuan et al., 2016; Sato et al., 2018). However, these studies were limited in scope, since only portions of the viral genomes collected at a single time point approximately 14-16 weeks post-infection were analyzed. Additionally, repeated exposures necessary to mimic serial transmission to a new set of human hosts were not explored. Addressing some of these limitations, our recent preliminary report described the in vivo passage of strain SIVcpzEK505, which is closely related to HIV-1 group N (Curlin et al., 2020). Full genome sequence analysis was performed at multiple time points during infection to understand the dynamic nature of the genetic changes related to viral adaptation. Many sequence variants were noted throughout the genome, some of which became the predominant genotype within the viral population. Building on these observations, we undertook a comprehensive approach to evaluate the SIVcpz infection and evolution toward HIV-1 in hu-mice. We focused on four main aspects to improve upon previous studies. First, we sequentially passaged the SIVcpz viruses in hu-mice for much longer periods in 2 consecutive passages in vivo spanning more than a year. Three different strains of SIVcpz (MB897, LB715, and EK505), from groups M and $\mathrm{N}$; respectively, were used. Second, viruses derived from the first passage were used to infect a second cohort of hu-mice to mirror serial transmission to a new human host. Third, we compared the viral loads and $\mathrm{CD}_{4}{ }^{+} \mathrm{T}$ cell decline between the first and second passage viruses to determine potential increased viral fitness and pathogenicity over time. Finally, we derived sequence data on the entire genomes at different time points to identify the sequential genetic changes resulting from human selective pressure. Our results showed that longer passaging allowed the virus to accumulate many significant mutations not seen in previous reports, and there was increased viral fitness for growth in the human surrogate system accompanied by numerous mutations that increased pathogenicity.

\section{RESULTS}

\section{Chimpanzee Native Viral Strains SIVcpzEK505, LB715, and MB897 Can Establish Productive Infection and Chronic Viremia in Humanized Mice}

To determine if SIVcpzEK505, MB897 and LB715 viruses of chimpanzee origin can establish productive infection, cohorts of five hu-HSC mice were inoculated with each of these and later serially passaged through different cohorts for a total of two generations (Figure 1). Plasma viral loads were assessed on a weekly basis using qRT-PCR with strain-specific primers and probes. Viral infection was evident within 14 days after the initial exposure with all the three SIVcpz progenitor 


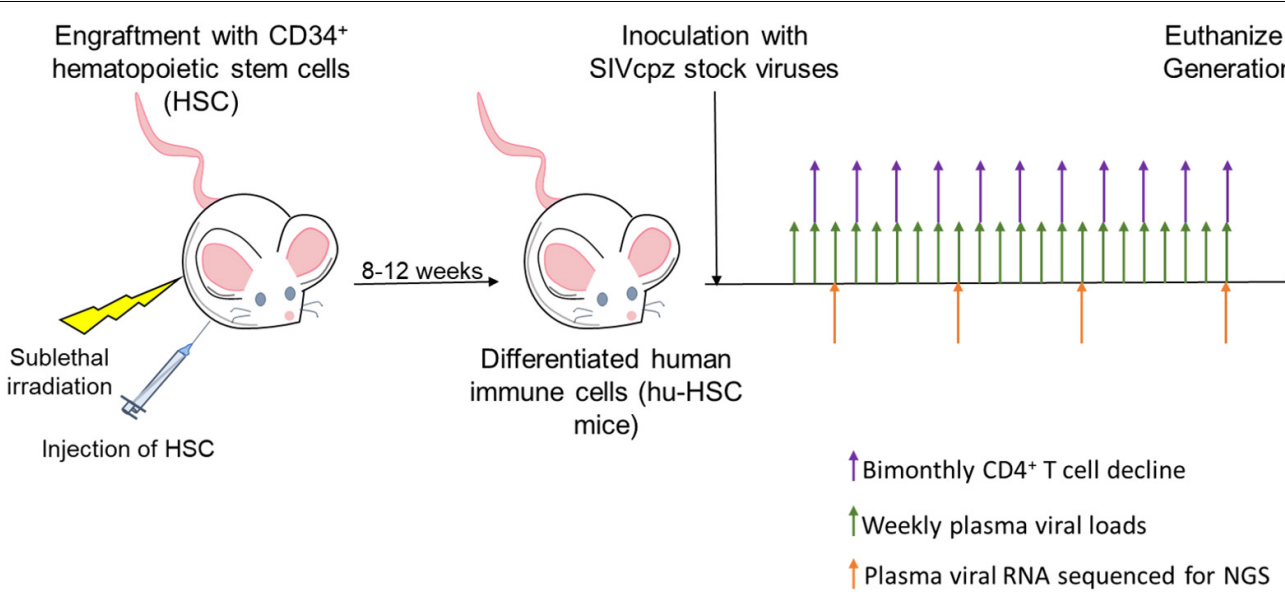

FIGURE 1 | Viral passaging experimental scheme. Neonatal mice were sublethally irradiated and injected intrahepatically with human CD34 ${ }^{+}$hematopoietic stem cells as described in the Section "Materials and Methods." Mice were screened 8-12 weeks post-reconstitution for human immune cell engraftment. Five hu-HSC mice with high human hematopoietic cell engraftment levels from a single cohort were infected intraperitoneally (i/p) with either cell-free SIVcpzEK505, LB715, or MB897 and monitored for plasma viral loads on a weekly basis using qRT-PCR. CD4 ${ }^{+}$T cell decline was assessed bimonthly by flow cytometry and plasma viral RNA was used for Illumina-based deep sequencing at 3, 11, 19-, and 24-weeks post-inoculation. The infected hu-HSC mice were euthanized at around 24 weeks and the isolated, infected cells were subsequently injected intraperitoneally into five naïve hu-HSC mice to begin the second passage/generation.

strains demonstrating their ability to productively infect human cells in vivo (Figure 2). Plasma viral loads in SIVcpzEK505infected hu-mice increased by 2-logs, peaking by 84 days postinoculation. In comparison, viral loads peaked 14 days sooner during the second passage and were 1-log higher indicating enhanced replicative fitness of the virus (Figure 2A). With the SIVcpzLB715 viral strain, first passage viral loads increased slowly increasing through 161 days ( $>10^{5}$ RNA copies $/ \mathrm{mL}$ ) post-inoculation (data not shown). However, the viral loads became more robust during the second generation peaking at 2-logs higher ( $>10^{7}$ RNA copies/mL) and 35 days earlier (112 days post-inoculation) (Figure 2B). Thus, the SIVcpzLB715 virus also appears to have gained growth fitness in human immune cells over time. While still successful in initiating infection, viral loads with the SIVcpzMB897 were found to be lower than the other strains tested. Viral loads stagnated slightly above the limit of detection for most of the first passage period and peaked ( $\geq 10^{4}$ RNA copies/mL) at 119 days postinoculation (Figure 2C). Unlike the other two strains above, the increase in second-generation plasma viral loads was slow for SIVcpzMB897 until around day 77. With the SIVcpzMB897 virus there was clearly a delay in viral adaptation to human immune cells during the first generation, and the viral growth appeared to evolve more rapidly during the middle of the second generation indicating eventual increased viral fitness. As expected, no plasma viral loads were detected in the control uninfected hu-HSC mice (data not shown). Previous data on HIV-1 BaL infections in hu-HSC mice showed that peak viral loads were reached at around 8 weeks post-inoculation (Berges et al., 2010). This is unlike the first generation of SIVcpz inoculations into hu-HSC mice where SIVcpzEK505, SIVcpzMB897 do not display peak viral loads till days 84 and 119, respectively, and SIVcpzLB715 does not peak during the first generation. In contrast, the viral loads peaked much earlier during the second generation, reflecting a greater adaptation toward human immune cells.

\section{Serial Passage of SIVcpz Viruses in Hu-Mice Leads to More Rapid CD4 ${ }^{+} \mathrm{T}$ Cell Decline}

$\mathrm{CD} 4^{+} \mathrm{T}$ cell decline is a key aspect of HIV infection (Aldrovandi et al., 1993; Baenziger et al., 2006; Berges et al., 2006; Denton and Garcia, 2011; Akkina, 2013). Accordingly, we followed the $\mathrm{CD}^{+} \mathrm{T}$ cell levels in SIVcpz infected mice over time. The baseline human $\mathrm{CD}^{+} \mathrm{T}$ cell levels prior to infection in all mice was greater than $50 \%$ of all human $\mathrm{CD}^{+}$cells. By the end of second generation, $\mathrm{CD}^{+} \mathrm{T}$ cell decline became apparent in mice infected with any of the three viruses tested $(p<0.001)$, with varying levels of decline depending on the virus (Figure 3). The only SIVcpz strain with a noticeable decline in $\mathrm{CD}^{+} \mathrm{T}$ cells during the first passage was SIVcpzEK505. This decline was observed beginning at day 14 and was maintained over the course of 6 months (Figure 3A). However, it became slightly more pronounced during the second passage wherein decline was also seen within 14 days post-inoculation and persisted throughout the course of infection. SIVcpzLB715 and SIVcpzMB897 strains displayed no notable CD4 ${ }^{+}$T cell decline during the first passage, but SIVcpzLB715 infected mice showed rapid $\mathrm{CD}^{+} \mathrm{T}$ cell loss 14 days post-inoculation during the second passage (Figure 3B). While the SIVcpzMB897 second generation showed a much less precipitous drop in $\mathrm{CD}_{4}^{+}$ $\mathrm{T}$ cells relative to the other SIVcpz strains, significant $\mathrm{CD} 4^{+}$ $\mathrm{T}$ cell decline was still observed 98 days post-inoculation (Figure 3C). Overall, these results showing rapid decline of $\mathrm{CD}^{+} \mathrm{T}$ cells during the second passage versus the first suggest enhanced viral adaptation and pathogenicity. More importantly, this data demonstrates that all three progenitor SIVcpz viruses 
A SIVcpzEK505

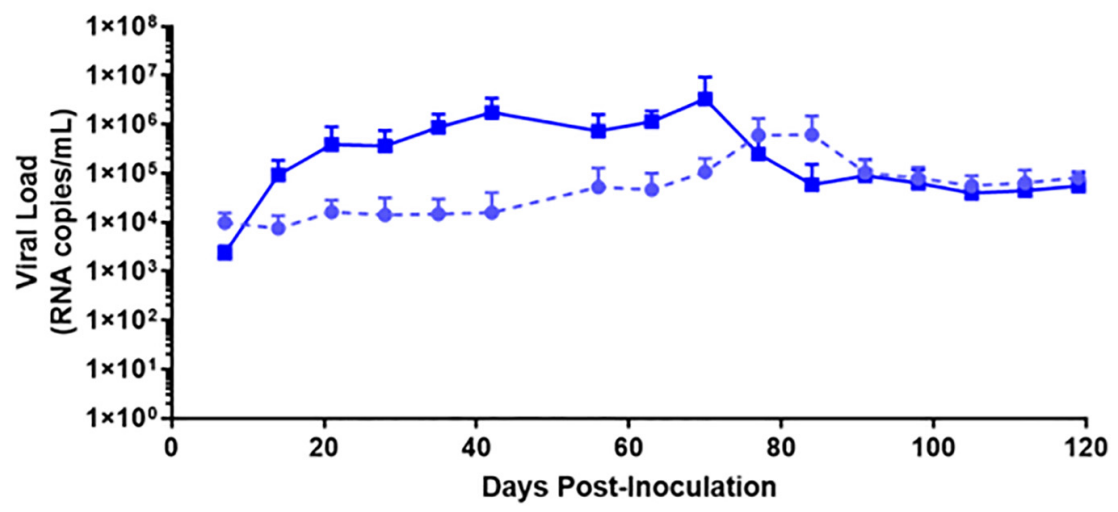

-๑. Infected Generation $1 \rightarrow$ Infected Generation 2

B SIVcpzLB715

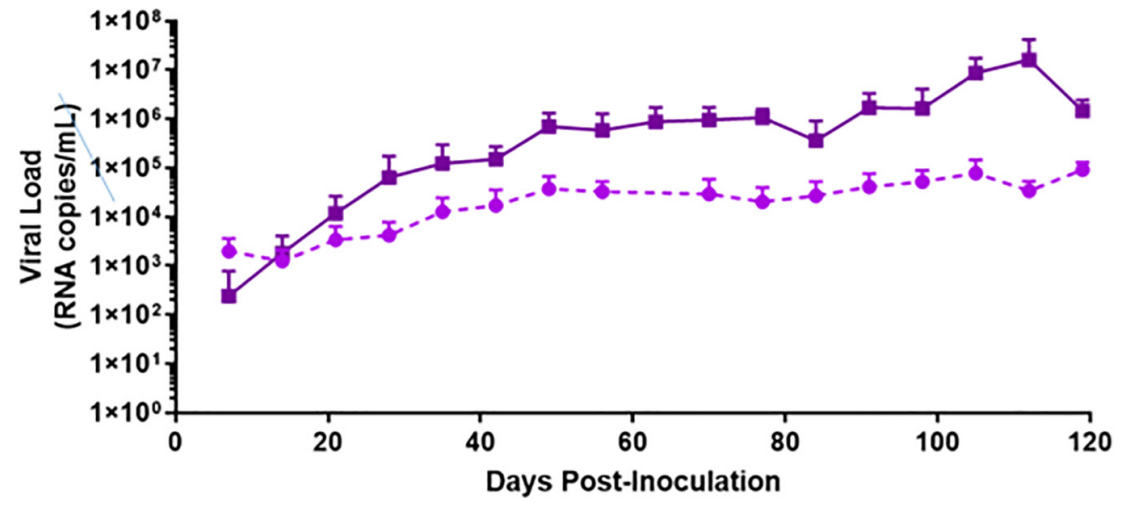

-๑. Infected Generation $1 \rightarrow$ Infected Generation 2

C SIVcpzMB897

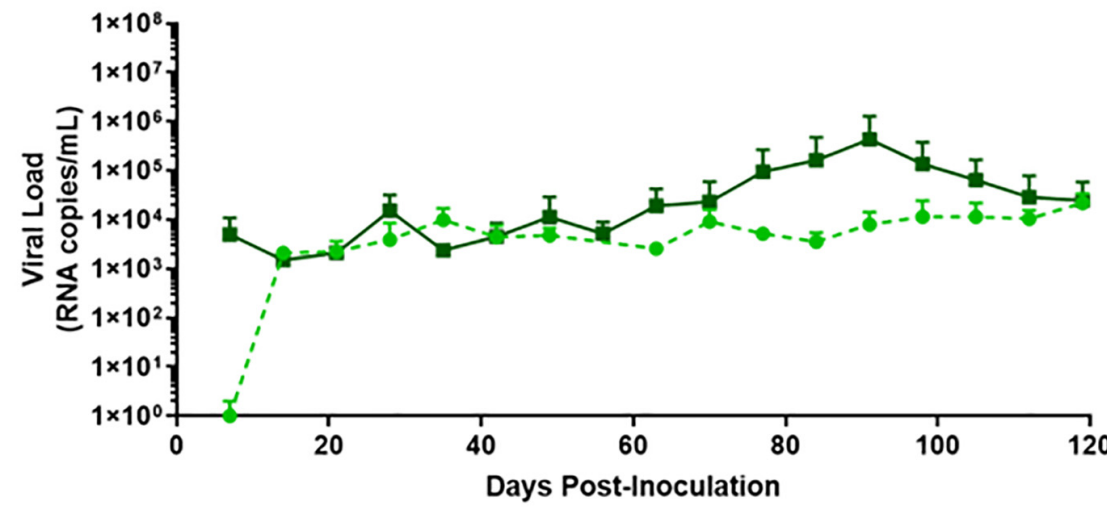

-•. Infected Generation $1 \rightarrow$ Infected Generation 2

FIGURE 2 | SIVcpz infection leads to detectable chronic viremia in humanized mice. (A) EK505, (B) LB715, and (C) MB897 first- and second-generation plasma viral loads. Five humanized mice were infected via i/p injections and the plasma viral loads were monitored by qRT-PCR on a weekly basis for the duration of the infection. No viral loads were detected in the uninfected control mice (data not shown). 
A

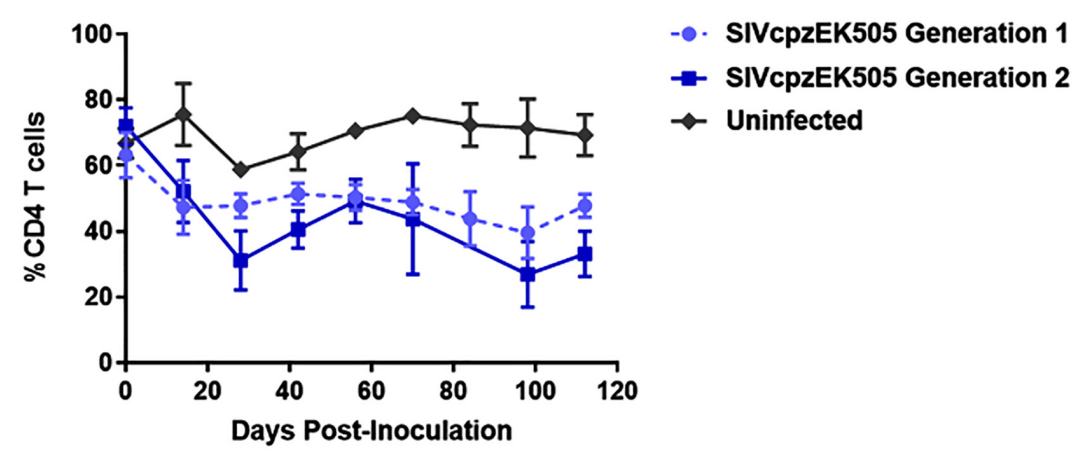

B

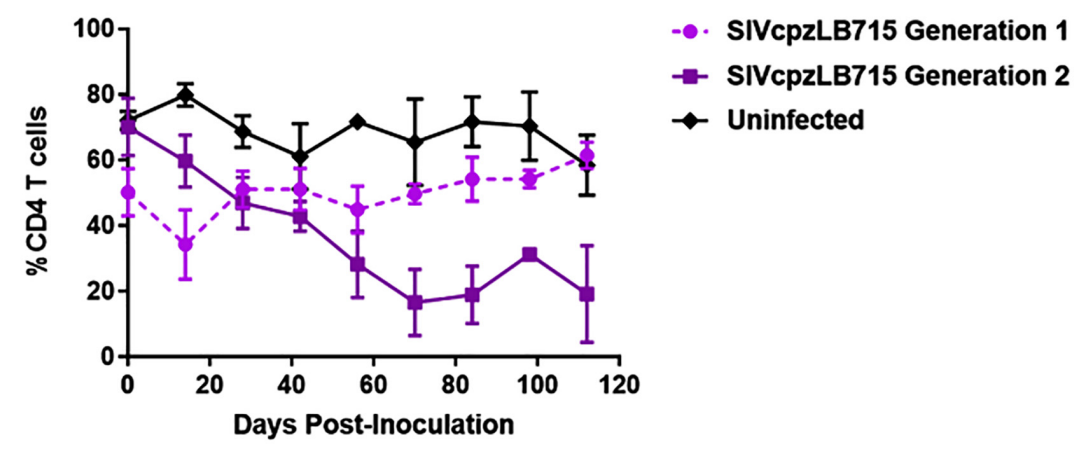

C

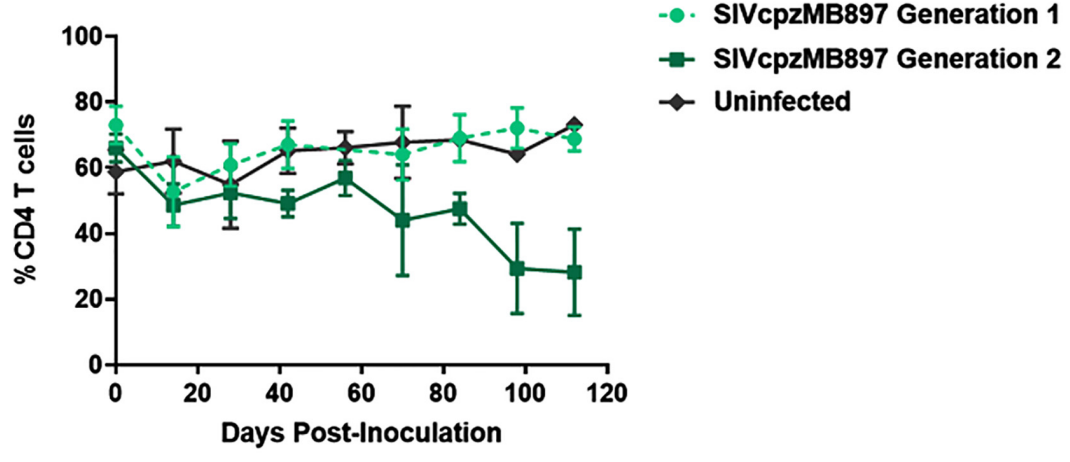

FIGURE 3 | SIVcpz infection leads to a gradual decline in CD4+ T cells in humanized mice. The (A) EK505, (B) LB715, and (C) MB897 first- and second-generation percentage of circulating $\mathrm{CD} 4^{+} \mathrm{T}$ cells relative to the total $\mathrm{CD} 45^{+} / \mathrm{CD} 3^{+}$cell populations were assessed on a bimonthly basis. Statistically significant depletion was seen in the second generation in infected hu-HSC mice relative to the uninfected control (two-tailed Student's $t$-test, $p<0.001$ ).

could establish productive viremia in hu-mice resulting in $\mathrm{CD}^{+} \mathrm{T}$ cell decline.

\section{SIVcpz Viruses Accumulate Adaptive Viral Genetic Changes During Long-Term Serial Passages in Hu-Mice}

Selection pressures imposed by the human immune cell environment in hu-mice are likely to promote adaptive changes in SIV progenitor viruses. This "genetic bottleneck" arises from both physical and immunological conditions that prevent most variants within the founder viral population from establishing infection within the human host (Joseph et al., 2015; Kariuki et al., 2017). The adaptive changes to viral fitness may be mediated through changes that improve viral entry, replication, epistasis, immune escape, host restriction factors and/or pathogenicity. Furthermore, novel mutations in the virus may arise due to these host selection pressures on genes such as vif, env, or nef that counteract host restriction factors like TRIM5 $\alpha$, APOBEC3 proteins and tetherin. To assess the genomic changes occurring in 
SIVcpz viral strains during passages in humanized mice through two generations, we utilized Illumina-based deep sequencing to identify possible signatures of viral adaptation. This was performed on the genomes of SIVcpzEK505, LB715 and MB897 clonal stock viruses used for initial infections and the viruses sampled at roughly 3, 11, 19-, and 24-weeks post-inoculation during the first and second passages. The consensus sequence of each viral stock was used as a reference point for aligning the sequence reads of passaged viruses at each time point. Variants that occurred at a frequency of greater than $1 \%$ of the population relative to the reference sequence were identified and then characterized as either synonymous or non-synonymous. We looked for potentially adaptive non-synonymous variants based on several criteria including: (1) variants that arose during the first passage and increased in frequency amongst the viral population over time and (2) variants that appeared in multiple mice within a given passage across both passages. All three SIVcpz strains tested gave rise to many new non-synonymous variants that increased in frequency across both serial passages which were absent in the original founder stock viral sequences (Tables 1-3). Nine variants matching this criterion were identified in
SIVcpzEK505 strain, seven in SIVcpzLB715, and with the greatest number of qualifying variants, 16, identified in SIVcpzMB897. All of these non-synonymous variants were absent in their respective stock virus population. The majority of substitutions occurring with at least $50 \%$ frequency at the last time point analyzed from the viruses sampled during the second passage were detected in the env gene, although other variants were found throughout the rest of the viral genome. SIVcpzMB897 had eleven nonsynonymous changes in env, two within gag and nef, and one within vif meeting these criteria (Table 3 ). Based on their relative size, the gag and pol genes displayed very few substitutions when compared to the env gene, which is consistent with the previous knowledge that the gag and pol genes are highly conserved amongst retroviruses. Several of these substitutions identified that arose during the first passage were maintained at almost $100 \%$ frequency during the second passage in both replicates of mice from which the sequenced viral samples were obtained (Supplementary Figure 1). Relatively few substitutions were seen in nef, vif, $v p u$, and $v p r$ genes in any of the SIVcpz strains tested which was surprising, given the role of these proteins in counteracting human restriction factors (Tables 1-3).

TABLE 1 | Amino acid substitutions resulting from candidate adaptive mutations in SIVcpzEK505 passaged virus.

\begin{tabular}{|c|c|c|c|c|c|c|c|c|c|}
\hline Protein & Position & $\begin{array}{c}\text { Stock } \\
\text { residue }^{a}\end{array}$ & $\begin{array}{l}\text { Variant } \\
\text { residue }^{b}\end{array}$ & $\begin{array}{l}\text { P1 endpoint } \\
\text { frequency }\end{array}$ & $\begin{array}{l}\text { P1 endpoint } \\
\text { frequency }^{d}\end{array}$ & $\begin{array}{l}\text { P2 endpoint } \\
\text { frequency }\end{array}$ & $\begin{array}{l}\text { P2 endpoint } \\
\text { frequency }^{f}\end{array}$ & SIV fraction ${ }^{g}$ & $\begin{array}{c}\text { HIV-1 } \\
\text { fraction }^{\mathrm{h}}\end{array}$ \\
\hline Gag & 35 & V & 1 & 0.88 & 0.14 & 1.00 & 1.00 & 0.00 & 0.04 \\
\hline Vif & 110 & $Y$ & $N$ & $0.00^{i}$ & $0.00^{i}$ & 0.72 & 0.30 & 0.00 & 0.00 \\
\hline Env & 291 & $K$ & $N$ & $0.00^{i}$ & $0.00^{i}$ & 0.85 & 0.76 & 0.78 & 0.96 \\
\hline Env & 402 & V & $\mathrm{G}$ & 0.56 & $0.00^{i}$ & 0.95 & 0.98 & 0.00 & 0.01 \\
\hline Env & 414 & $P$ & S & $0.00^{i}$ & $0.00^{i}$ & 0.90 & 0.64 & 0.00 & 0.04 \\
\hline Env & 611 & S & $\mathrm{T}$ & 0.16 & $0.00^{i}$ & 0.81 & 0.94 & 0.61 & 0.95 \\
\hline Env & 616 & $\mathrm{G}$ & $E$ & $0.00^{i}$ & $0.00^{i}$ & 0.87 & 0.78 & 0.04 & 0.07 \\
\hline Env & 668 & $\mathrm{R}$ & $\mathrm{K}$ & 0.30 & $0.00^{i}$ & 0.88 & 0.31 & 0.04 & 0.02 \\
\hline Env & 703 & $E$ & K & 0.60 & 0.39 & 1.00 & 0.99 & 0.00 & 0.00 \\
\hline
\end{tabular}

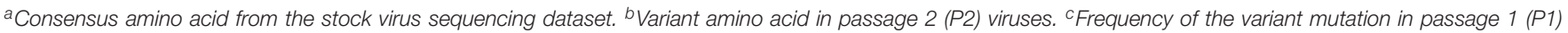

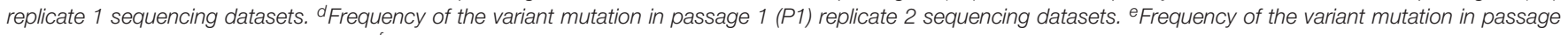

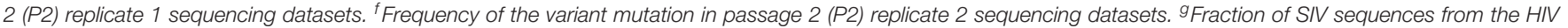

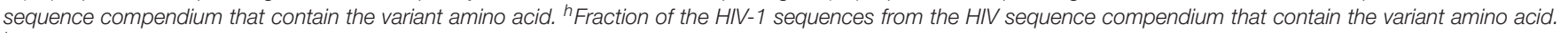
iThe 0 indicates below the limit of detection (1\%) of our variant identification pipeline.

TABLE 2 | Amino acid substitutions resulting from candidate adaptive mutations in SIVcpzLB715 passaged virus.

\begin{tabular}{|c|c|c|c|c|c|c|c|c|c|}
\hline Protein & Position & $\begin{array}{c}\text { Stock } \\
\text { residue }^{a}\end{array}$ & $\begin{array}{l}\text { Variant } \\
\text { residue }^{\mathrm{b}}\end{array}$ & $\begin{array}{l}\text { P1 endpoint } \\
\text { frequencyc }\end{array}$ & $\begin{array}{l}\text { P1 endpoint } \\
\text { frequency }^{d}\end{array}$ & $\begin{array}{l}\text { P2 endpoint } \\
\text { frequency }\end{array}$ & $\begin{array}{l}\text { P2 endpoint } \\
\text { frequency }^{f}\end{array}$ & SIV fraction ${ }^{g}$ & $\begin{array}{c}\text { HIV-1 } \\
\text { fraction }^{h}\end{array}$ \\
\hline Pol & 329 & $E$ & K & $0.00^{i}$ & $0.00^{i}$ & 0.84 & 0.55 & 0.00 & 0.01 \\
\hline Vpr & 68 & $L$ & $\mathrm{P}$ & $0.00^{i}$ & $0.00^{i}$ & 0.85 & 0.97 & 0.00 & 0.00 \\
\hline Vpu & 45 & $R$ & $\mathrm{~T}$ & $0.00^{i}$ & $0.00^{i}$ & 0.66 & 0.79 & 0.00 & 0.00 \\
\hline Env & 523 & $E$ & Q & $0.00^{i}$ & $0.00^{i}$ & 0.50 & 0.76 & 0.00 & 0.00 \\
\hline Env & 800 & $G$ & $\mathrm{R}$ & 0.55 & 0.25 & 1.00 & 1.00 & 0.00 & 0.00 \\
\hline Env & 841 & G & $\mathrm{R}$ & $0.00^{i}$ & $0.00^{i}$ & 0.47 & 0.60 & 0.00 & 0.00 \\
\hline Nef & 36 & $D$ & $N$ & $0.00^{i}$ & $0.00^{i}$ & 0.83 & 0.93 & 0.00 & 0.00 \\
\hline
\end{tabular}

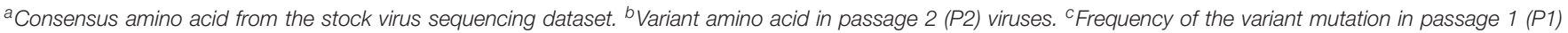

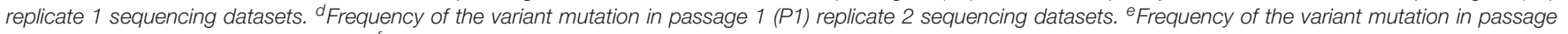

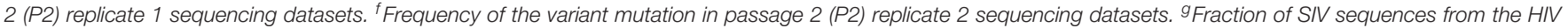

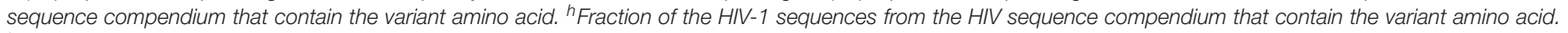

${ }^{i}$ The 0 indicates below the limit of detection (1\%) of our variant identification pipeline. 
TABLE 3 | Amino acid substitutions resulting from candidate adaptive mutations in SIVcpzMB897 passaged virus.

\begin{tabular}{|c|c|c|c|c|c|c|c|c|c|}
\hline Protein & Position & $\begin{array}{c}\text { Stock } \\
\text { residue }^{a}\end{array}$ & $\begin{array}{l}\text { Variant } \\
\text { residue }^{b}\end{array}$ & $\begin{array}{l}\text { P1 endpoint } \\
\text { frequency }\end{array}$ & $\begin{array}{l}\text { P1 endpoint } \\
\text { frequency }^{d}\end{array}$ & $\begin{array}{l}\text { P2 endpoint } \\
\text { frequency }\end{array}$ & $\begin{array}{l}\text { P2 endpoint } \\
\text { frequency }^{f}\end{array}$ & SIV fraction ${ }^{g}$ & $\begin{array}{c}\text { HIV-1 } \\
\text { fraction }^{h}\end{array}$ \\
\hline Gag & 35 & V & I & $0.00^{i}$ & $0.00^{i}$ & 0.99 & 0.64 & 0.00 & 0.04 \\
\hline Gag & 105 & $E$ & $\mathrm{~K}$ & $0.00^{i}$ & $0.00^{i}$ & 0.90 & 0.74 & 0.69 & 0.02 \\
\hline Vif & 47 & $E$ & $\mathrm{~K}$ & 0.88 & 1.00 & 1.00 & 1.00 & 0.00 & 0.01 \\
\hline Env & 149 & $N$ & Y & 0.54 & 0.68 & 0.97 & 0.75 & 0.00 & 0.01 \\
\hline Env & 346 & $R$ & Q & $0.00^{i}$ & 0.50 & 1.00 & 0.99 & 0.00 & 0.02 \\
\hline Env & 351 & S & $\mathrm{N}$ & $0.00^{i}$ & 0.28 & 1.00 & 0.99 & 0.04 & 0.00 \\
\hline Env & 413 & $\mathrm{G}$ & $\mathrm{R}$ & 0.83 & 0.64 & 0.99 & 0.99 & 0.00 & 0.00 \\
\hline Env & 413 & $\mathrm{G}$ & $E$ & 0.14 & 0.66 & 0.99 & 0.99 & 0.00 & 0.01 \\
\hline Env & 414 & K & $\mathrm{R}$ & 0.33 & $0.00^{i}$ & 0.84 & 0.91 & 0.17 & 0.30 \\
\hline Env & 442 & $N$ & I & $0.00^{i}$ & 0.51 & 0.99 & 0.99 & 0.04 & 0.06 \\
\hline Env & 475 & $\mathrm{~L}$ & V & 0.08 & 0.60 & 0.74 & 0.52 & 0.87 & 0.50 \\
\hline Env & 596 & $K$ & $N$ & 0.49 & 0.07 & 0.40 & 0.87 & 0.26 & 0.00 \\
\hline Env & 702 & $\mathrm{~F}$ & $L$ & 0.17 & 0.10 & 0.90 & 0.61 & 0.04 & 0.29 \\
\hline Env & 838 & G & $S$ & 0.12 & 0.94 & 1.00 & 1.00 & 0.00 & 0.00 \\
\hline Nef & 126 & $N$ & $K$ & 0.89 & 0.08 & 0.34 & 0.74 & 0.00 & 0.00 \\
\hline Nef & 163 & V & $M$ & 0.57 & 0.22 & 0.99 & 0.98 & 0.00 & 0.00 \\
\hline
\end{tabular}

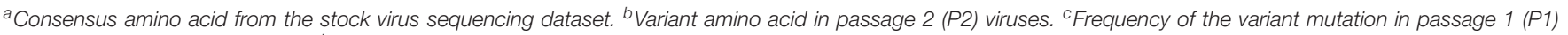

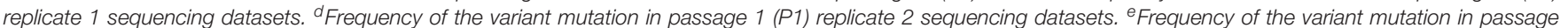

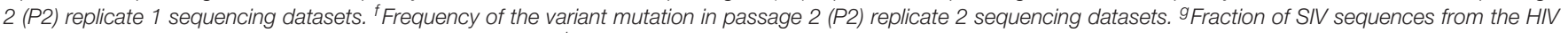

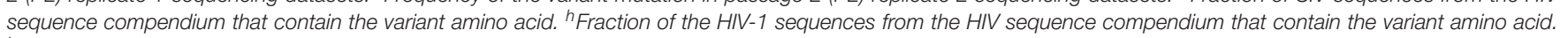
iThe 0 indicates below the limit of detection (1\%) of our variant identification pipeline.

Utilizing the sequence alignments in the HIV Sequence Compendium, we next sought to assess whether or not each nonsynonymous variant observed produced a more "HIV-1-like" or "SIV-like" virus (Foley et al., 2017). We determined if a variant amino acid detected is present in a higher fraction of HIV-1 or SIV sequences (Tables 1-3). Two amino acid substitutions identified in Env for both SIVcpzMB897 (K414R and F702L) and SIVcpzEK505 (K291N and S611T) strains were found in a higher fraction of HIV-1 sequences than in those of SIVs', indicating a change more representative of HIV than SIV. Interestingly, with the SIVcpzLB715 strain, the detected substitutions were neither more "SIV-like" nor "HIV-1-like" in nature. Additional passages in vivo of these SIVcpz viruses during a much longer time span may be necessary to observe the evolution toward HIV-1.

\section{DISCUSSION}

Here, we evaluated both the genetic and phenotypic changes that occurred during the evolution of progenitor SIV viruses toward HIV-1 using a humanized mouse model. In the first set of experiments, clonal stocks of three NHP SIV strains SIVcpz LB715, MB897 and EK505 belonging to groups $M$ and $\mathrm{N}$; respectively, were inoculated into hu-HSC mice to determine whether they can establish infection in the human surrogate host and affect $\mathrm{CD}^{+}{ }^{+} \mathrm{T}$ cell levels. Later, the viruses were serially passaged in different cohorts of hu-mice to mimic serial human transmission and evaluate the potential increase in viral fitness and pathogenicity. While no animal model is perfect in fully mimicking the human system, the hu-HSC mouse model used here has several advantages over using human PBMC in in vitro experiments. These include, provision of a physiologic in vivo environment, immune pressures and prolonged time frame like 6 months or longer for serial viral propagation/adaptation. Each of the viral strains was able to infect hu-mice validating that SIVs originating from chimpanzees are capable of readily infecting human hosts as might have happened in the past. We followed the kinetics of viremia for several months in infected hu-mice and found there was a slow but steady increase in viremia over time (Figures 2A-C) during the first passage with each of the viral strains used. When the viruses from the first passage were used to infect new cohorts of hu-mice, viremia levels rapidly increased with the viral load set points being relatively higher than in the first passage. This showed that the SIVcpz strains were adapting to propagate better in the hu-mouse system as a consequence of increased fitness. Since helper $\mathrm{CD}^{+} \mathrm{T}$ cell decline is a hallmark of HIV-1 infection that results in immunosuppression, we also assessed this trait between the first and second passage of the hu-mouse adapted SIV strains. Whereas a slower pace of $\mathrm{CD}_{4}^{+}$ $\mathrm{T}$ cell decline was noted during the first passage with all three viral strains over time, the cell decline became more pronounced and rapid during the second passage (Figures 3A-C). Both the increased amplitude of the viral loads and faster pace of $\mathrm{CD}^{+} \mathrm{T}$ cell decline seen with each strain during the second passage is indicative of viral evolution resulting in increased human pathogenicity.

While all the three SIVcpz viral strains tested above were capable of initiating infection in hu-mice, the SIVcpzMB897 strain displayed less robust viral loads than the others during the first passage. This contrasts with the conclusions of Sato et al. (2018) which reported that SIVcpzMB897 was more preadapted toward human immune cells compared to the 
other SIVcpz strains. Our findings are further supported by the longer timeframe required for noticeable $\mathrm{CD}^{+} \mathrm{T}$ cell decline by SIVcpzMB897 when compared to SIVcpzLB715 and SIVcpzEK505 strains. Furthermore, sequence data showed a greater number of variants in SIVcpzMB897 relative to the other two SIVcpz strains during the serial passage indicating it requires further adaptation for efficient propagation in humans.

Based on sequence differences documented between SIVs and HIVs, it is clear that numerous genetic changes must have taken place in the progenitor SIV to give rise to the four known HIV-1 lineages (Keele et al., 2006; Van Heuverswyn et al., 2006; Sharp and Hahn, 2011; Hemelaar, 2012; Sauter and Kirchhoff, 2019). During this process, the progenitor SIVs had to evade or adjust to a number of native human cell barriers capable of interference with many steps in the viral life cycle. In addition to cell surface receptors involved in viral attachment, other barriers consist of restriction factors. These currently include, but are not limited to, TRIM5 $\alpha$, APOBEC3 proteins, SAMHD1, SERINC3/5, and tetherin which function by various mechanisms (Sheehy et al., 2002; Sayah et al., 2004; Stremlau et al., 2004; Neil et al., 2008; Le Tortorec and Neil, 2009; Berger et al., 2011; Goldstone et al., 2011; Serra-Moreno and Evans, 2012; McNatt et al., 2013; Etienne et al., 2015; Rosa et al., 2015; Usami et al., 2015; Ghimire et al., 2018; Meyerson et al., 2018). The list of restriction factors is continually expanding pointing to many other yet undiscovered intrinsic host defense mechanisms (Ghimire et al., 2018). These factors impose a strong selective pressure on non-native viruses to acquire adaptive changes. To identify potential genetic changes, we analyzed whole viral genomes from the first and second passages at different time points with a focus on non-synonymous genetic changes. Viral variants that increased in population frequency from the first to the second generation are presented in Figure 4. Some variants occurring in low frequency at the early time points became more prominent/dominant during later time points and became fixed (Tables 1-3). We also noticed several variants of transient nature that were seen at early time points which disappeared subsequently (data not shown).

Among the recent reports on SIV infection and evolution in humanized mice (Yuan et al., 2016, 2018; Sato et al., 2018), only limited regions of the viral genomes were evaluated by sequence analysis in one report and only variants from shortterm infection (14-16 weeks of infection) at a single terminal time point were analyzed in all three studies (Yuan et al., 2016, 2018; Sato et al., 2018). To extend and improve upon these early studies and increase the breadth of analysis, here we examined the viral variants sampled at multiple time points during a much longer period spanning more than a year. Sequential passages performed in different cohorts of hu-mice also allowed the recapitulation of serial viral transmission and subsequent spread to a new human host. Not surprisingly, numerous changes could be identified throughout the viral genome beyond the two mutations described in the envelope gene in the studies mentioned above (Yuan et al., 2016; Sato et al., 2018).

Among the many non-synonymous changes seen, of note is the V35I substitution in Gag. This arose independently in both the EK505 (Group N) and MB897 (Group M) strains. While its exact function needs to be determined, that it arose in two divergent progenitor viral strains points to an essential role for it in human immune cell adaptation. However, this change was not seen in the LB715 strain (also group M, like MB897). More passages of LB715 may be needed for it to appear at a later time point or alternatively, other mutations in the vicinity might be playing a compensatory role. We also looked for the potential emergence of an important Gag M30K/R substitution, described previously in elegant studies by Wain et al. (2007), Sharp and Hahn (2011), and Bibollet-Ruche et al. (2012) detailing the origins of HIVs from NHP viruses. This mutation was shown to be highly conserved in HIV-1, but absent in most SIVs, suggesting it being a species-specific signature. However, it was not detected in our present studies. Corroborating our findings here, results from recent reports on SIVcpzMB897, SIVcpzEK505 and other chimpanzee-derived strains passaged in hu-mice also did not detect this Gag M30K/R substitution (Yuan et al., 2016; Sato et al., 2018). Thus, this substitution may not be as critical as previously thought for human cell adaptation, at least to overcome intracellular barriers during the early stages of human propagation. Supporting this hypothesis is the observation by Sato et al. (2018) that introduction of this substitution into SIVcpzMB897 strain did not augment viral fitness and replication kinetics in hu-mice. Alternatively, another variant the V35I substitution namely seen here may be playing an equivalent role similar to the Gag M30K/R substitution.

The viral envelope protein is involved in host cell receptor binding and dictates the efficiency of initial viral host cell interactions. Importantly, a non-synonymous change in Env identified in strain MB897 in two previous reports and found to improve viral pathogenicity, the Env G413R(/E) mutation, was also detected in our studies (Yuan et al., 2016; Sato et al., 2018). This variant appeared at 11 weeks post-inoculation and later became fixed. Its detection in three independent studies from different laboratories makes a compelling case for its critical nature in the SIVcpzMB897 strain's adaptation to human cells. During the course of our two serial passages, we also observed two additional mutations of significance, namely, the Env N442I and K414R substitutions in the MB897 strain. These increased in frequency during the first passage and remained stable through the second passage. Env N442I is located adjacent to a wellcharacterized CD4 binding site in the V5 loop of envelope and potentially aids in facilitating viral attachment (Xiang et al., 2002; Chen et al., 2005; Decker et al., 2005; Yu et al., 2010). Another mutation, the Env K414R change, located in the CD4 binding site itself appeared later and began to increase in frequency during the second passage. It is possible that the initial adjacent mutations "prime" the site itself to better accommodate more drastic changes to the site, which may explain the delayed appearance of the Env K414R mutation relative to the others. The other two SIV strains tested also showed adaptive changes in receptor interacting sites. In SIVcpzEK505, an Env variant V402G in the CD4 binding site appeared during the late first passage (week 19) and eventually made up $100 \%$ of the viral population by the second passage. Another substitution directly adjacent to the chemokine binding site, the Env P414S, was detected at the beginning of the second passage (Xiang et al., 2002; Chen et al., 2005; Decker et al., 2005; Yu et al., 2010). These changes may 


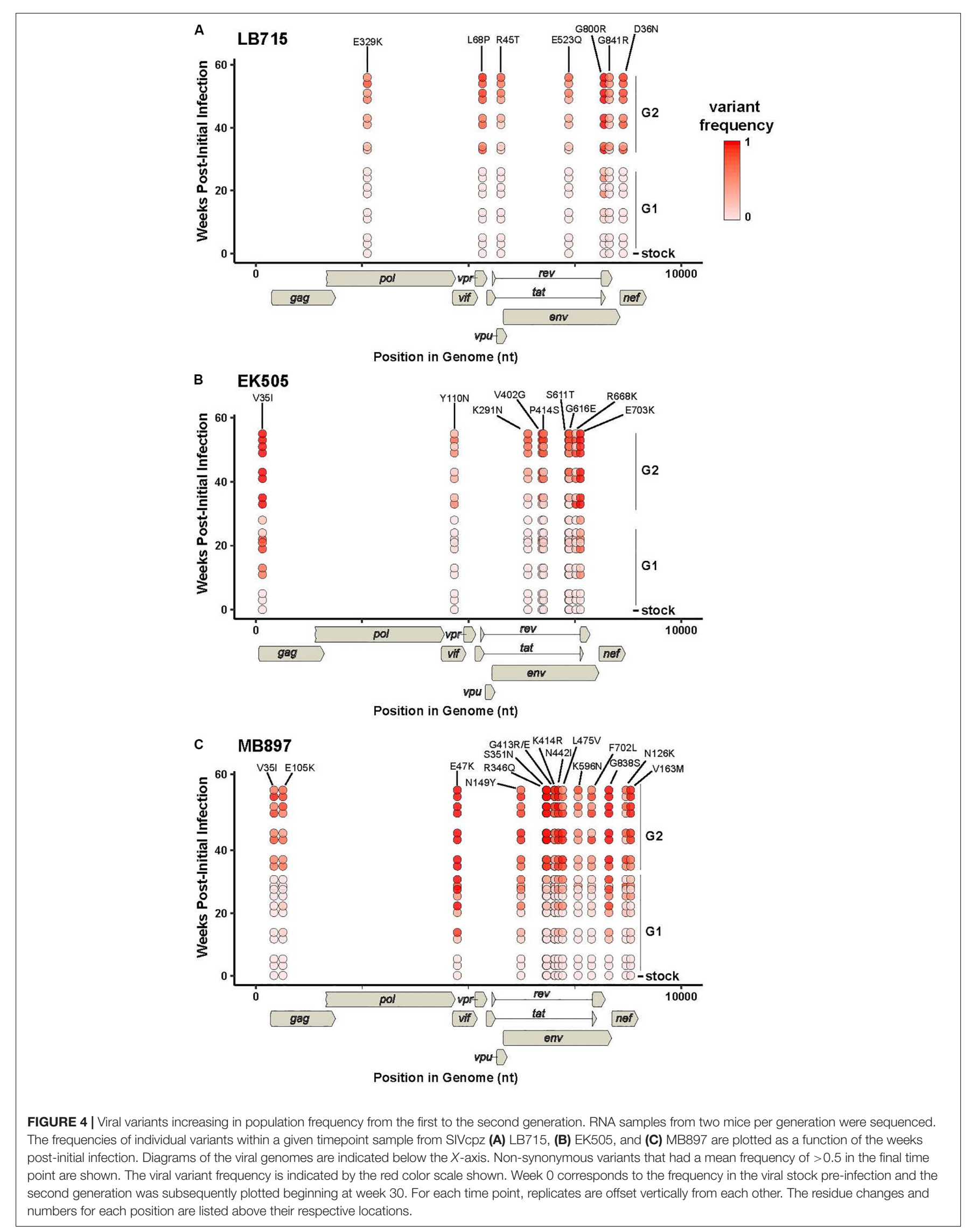


have contributed to increased pathogenicity of SIVcpzEK505 as evidenced by both increased viral loads and more rapid CD4 ${ }^{+}$ $\mathrm{T}$ cell decline that commenced at the beginning of the second passage. Similarly, the Env E523Q mutation seen in SIVcpzLB715 is located in the CD4 binding site and appeared during the second passage (Xiang et al., 2002; Chen et al., 2005; Decker et al., 2005; Yu et al., 2010). By evolving the CD4 and chemokine receptor binding sites, these viruses may be gaining improved engagement of the cell receptors and an overall higher fitness to the human host.

Several of the variants we detected did not appear until relatively late during the first hu-mouse passage (19-24 weeks post-infection) and did not become predominant until the second passage (Supplementary Figure 1). A number of other mutations were shown to arise at single time points and then disappear entirely indicating their transient nature and irrelevance in the context of viral evolution. These observations underscore the importance of longer in vivo viral passages and serial propagation to ascertain viral variants at multiple time points as they arise.

In addition to incompatible cell receptors barriers for viral entry, other major barriers for virus transmission is the myriad of host restriction factors such as TRIM $5 \alpha$, APOBEC3 proteins and tetherin. One of the functions of $\mathrm{Vpu}$ is to overcome tetherin. In some cases only a few genetic changes appear to be necessary to allow chimpanzee $\mathrm{Vpu}$ to be effective against human tetherin (Lim et al., 2010; Vigan and Neil, 2010; Kluge et al., 2013; Sauter and Kirchhoff, 2019). While we have not yet detected any variants directly connected to the known human restriction factors at these serial passage levels, several other significant variants with increased frequency are noted as shown in Tables 1-3, including the Vpu R45T substitution identified in SIVcpzLB715. We also identified two amino acid substitutions in both SIVcpzEK505 Y110N and SIVcpzMB897 E47K, which appeared fixed by the end of the second generation. Both of these variants were below the limit of detection in the starting stock viruses (Tables 1, 3). Additional Vif variants may continue to arise over repeated passaging in our humanized mouse model to further confirm candidate adaptive mutations that may contribute to counteracting the action of APOBEC3G (or APOBEC3 proteins) in human immune cells. These mutations and others need to be further characterized to determine their role in viral adaptation, pathogenesis and fitness. These mutation and others need to be further analyzed to determine their role in viral adaptation, pathogenesis and fitness. Two variants in Pol and Vpr, Pol E329K and Vpr L68P, that were identified in viral strain SIVcpzLB715 arose at the beginning of the second passage and then were maintained in over half of the viral population indicating their potential role for viral adaptation (Table 2). The Pol E329K mutation is located in the p51 reverse transcriptase subunit of the viral polymerase (Nagata et al., 2017) and the Vpr L68P substitution is found within the "LxxLL" motif (Kino et al., 1999; Kamata and Aida, 2000; Sherman et al., 2000; Kogan and Rappaport, 2011; Gonzalez, 2017). While, the function of the Pol E329K substitution is still unknown, the "LxxLL" motif is required for glucocorticoid-dependent activation of transcription to maintain resting and stress-related homeostasis as well as being directly implicated in the nuclear localization of the PIC complex (Kino et al., 1999; Kamata and Aida, 2000; Sherman et al., 2000; Gonzalez, 2017). This same mutation (Vpr L68P) was also identified as a proline (LxxLP) in HIV-1 NL4-3. This proline change has been noted in a participant sample but was not tested experimentally to confirm its effect on viral pathogenicity. However, previous studies have found that direct mutation of this residue leads to rapid progression of AIDS (Sherman et al., 2000; Gonzalez, 2017). Additionally, it is possible that some of these mutations may confer no additional fitness, but rather become fixed stochastically. However, the persistence of these mutations across multiple generations and immune environments make it unlikely that non-favorable mutations would increase in frequency within the viral population overtime. Further studies and passages of these viruses are needed to determine the direct function of these amino acid substitutions.

An important question is whether the non-synonymous variants seen here during serial passages in a human surrogate system reflect evolution toward HIV-1 as a whole or singly align with those of existing variants in the SIV genomes. Sequence alignments were done using the HIV Sequence Compendium (Foley et al., 2017). Specifically, we assessed whether the observed amino acid variants are present in a higher fraction of HIV1 sequences than in SIV sequences. Most of the mutations we identified were present in very few SIV or HIV strains. However, two variants, Env K291N and Env S611T observed in the EK505 progenitor strain were found approximately $20 \%$ more frequently in HIV sequences than in SIV (Foley et al., 2017). However, these substitutions have no known function. The variant Env F702L seen in MB897 strain is located in the gp41 of Env adjacent to the Kennedy Epitope but no function is known (Steckbeck et al., 2011). None of the variants seen in the LB715 strain appeared to be common in either SIV or HIV strains. Several other variants seen were found be to more common in SIVs than in HIVs though the functionality of these changes remains unclear.

In summary, our results showed that HIV progenitor SIVcpz viral strains, from the pandemic Group M (MB897 and LB715) and non-pandemic Group N (EK505), can readily infect humanized mice leading to chronic viremia and upon further passage, marked $\mathrm{CD}^{+}{ }^{+} \mathrm{T}$ cell decline. These findings reinforce the utility of the hu-mouse human surrogate system in modeling cross-species transmission and viral adaptation. Whereas the non-synonymous sequence changes seen in the CD4 and chemokine receptor binding Env regions of these evolving viruses show one aspect of enhancing viral affinity and fitness to the human host, other notable changes might contribute to other steps in viral replication. While the sequences of the variant viruses at the second passage spanning several months do not appear to be more like HIV, the SIV progenitors have responded to the selection pressure imposed by the in vivo human system by acquiring sequence changes. It remains to be seen if additional passages in hu-mice would impose further selection giving rise to viral strains more like HIV-1. Nevertheless, the ease with which these viruses infected humanized mice is remarkable, suggesting that many SIV strains currently circulating in the wild continue to pose a significant zoonosis risk to the human and have the 
potential to generate dangerous new HIV strains in the future. With the knowledge gained on how SIVcpz first adapted to human immune cells and gained virulence we will be able to better predict if, and how, other NHP derived lentiviruses may cross over into humans in the future.

\section{MATERIALS AND METHODS}

\section{Preparation of the SIVcpz Viral Stocks}

In order to generate virus, full-length infectious molecular clones of pSIVcpzMB897, pSIVcpzLB715, and pSIVcpzEK505 were transfected into $293 \mathrm{~T}$ cells. Briefly, $15 \mu \mathrm{g}$ of plasmid DNA and $30 \mu \mathrm{l}$ of TurboFect transfection reagent (Thermo Fisher Scientific, Waltham, MA, United States) was diluted in serumfree DMEM to transfect 293T cells seeded in a T150 flask. At $48 \mathrm{~h}$, virus supernatants were harvested and clarified by low speed centrifugation. To concentrate the viral inoculum, clarified supernatant was ultracentrifuged on a $20 \%$ sucrose $(\mathrm{w} / \mathrm{v})$ cushion in Ultra-Clear tubes (Beckman Coulter, Pasadena, CA, United States). Ultracentrifugation was conducted utilizing a L8-70M ultracentrifuge and SW 28 Ti rotor (Beckman Coulter, Pasadena, CA, United States) at $27,000 \mathrm{rpm}$ for $2 \mathrm{~h}$ at $4^{\circ} \mathrm{C}$. Supernatant was decanted and pelleted virus was resuspended gradually on ice for $20 \mathrm{~min}$ in serum-free DMEM. Resuspended, cell-free virus was used to directly infect humanized mice, as described below, and an aliquot was used for titration on TZMbl cells. Briefly, virus was serially diluted from $10^{-1}$ to $10^{-6}$ and used to inoculate TZM-bl reporter cells. At $48 \mathrm{~h}$, media was removed, cells were washed with PBS and the monolayer was fixed using $1 \%$ formaldehyde- $0.2 \%$ glutaraldehyde in PBS for $5 \mathrm{~min}$. Cells were washed and incubated in a solution of $4 \mathrm{mM}$ potassium ferrocyanide, $4 \mathrm{mM}$ potassium ferricyanide, $4 \mathrm{mM}$ magnesium chloride and $0.4 \mathrm{mg} X$-gal per $\mathrm{ml}$, for $2 \mathrm{~h}$ at $37^{\circ} \mathrm{C}$. The reaction was stopped and the tissue culture infectious dose $\left(\mathrm{TCID}_{50}\right)$ per $\mathrm{ml}$ was calculated to determine virus titer (Derdeyn et al., 2000; Wei et al., 2002).

\section{Generation of Humanized Mice}

Humanized mice were prepared using fetal liver-derived CD34 cells that were isolated, column purified (Miltenyi Biotec, San Diego, CA, United States), cultured and assessed for purity utilizing flow cytometry as previously described (Akkina et al., 1994; Bai et al., 2000; Veselinovic et al., 2016; Schmitt et al., 2017). Preconditioned by irradiation with $350 \mathrm{rads}$, neonatal $\mathrm{Balb} / \mathrm{c} \mathrm{Rag}^{-/-} \gamma \mathrm{c}^{-/-}$or $\mathrm{Balb} / \mathrm{c}$ $\mathrm{Rag}^{-/-} \gamma \mathrm{c}^{-/-}$mice were intrahepatically injected with $0.5-$ $1 \times 10^{6}$ human $\mathrm{CD}_{4} 4^{+}$cells per mouse (Berges et al., 2008; Veselinovic et al., 2016). Human cell engraftment was then determined by flow cytometry in transplanted mice at $10-12$ weeks post-reconstitution by collecting peripheral blood. The red blood cells were lysed using the Whole Blood Erythrocyte Lysing Kit according to the manufacturer's instructions (R\&D Systems, Minneapolis, MN, United States). Fractioned white blood cells were stained with mouse antihuman CD45 FITC (eBioscience), CD3 PE (eBioscience) and $\mathrm{CD} 4 \mathrm{PE} / \mathrm{Cy} 5$ (eBioscience) for flow cytometry in order to confirm human cell engraftment (Berges et al., 2006; Veselinovic et al., 2016; Schmitt et al., 2017). All mice were maintained at the Colorado State University Painter Animal Center. The studies conducted in this publication have been reviewed and approved by the CSU Institutional Animal Care and Use Committee.

\section{SIVcpz Infection of Humanized Mice and Viral Load Determination by qRT-PCR}

Mice with high $\left(>60 \% \mathrm{CD}^{+} 5^{+},>50 \% \mathrm{CD}^{+}\right)$human hematopoietic cell engraftment levels were used. At 16 weeks post-engraftment, $200 \mu \mathrm{l}$ of cell-free SIVcpzMB897 $\left(2 \times 10^{5}\right.$ TCID $\left._{50}\right)$, SIVcpzEK505 $\left(3.2 \times 10^{5}\right.$ TCID $\left._{50}\right)$, and SIVcpzLB715 $\left(2 \times 10^{5} \mathrm{TCID}_{50}\right)$ were used to inject five hu-HSC mice intraperitoneally (i/p). To assess plasma viral loads, peripheral blood was collected weekly by tail vein puncture using nonheparinized capillary tubes and transferred immediately to EDTA-containing vacutainer tubes (BD Biosciences, San Jose, CA, United States). The peripheral blood was mixed with PBS for a final volume of $150 \mu \mathrm{l}$ and centrifuged for $5 \mathrm{~min}$ at $400 \times \mathrm{g}$. Plasma was collected, and viral RNA was extracted from the plasma using the E.Z.N.A. Viral RNA kit according to the manufacturer's instructions (OMEGA Bio-Tek, Norcross, GA, United States). Viral loads were determined using the iScript One-Step RT-PCR kit with SYBR green and the manufacturer's instructions (Bio-Rad, Hercules, CA, United States). Virus-specific primers were designed based on a conserved region in the LTR of SIVcpzEK505, SIVcpzMB897 and SIVcpzLB715 (GenBank accession numbers: DQ373065, EF535994.1, and KP861923.1, respectively). The primers designed for qRT-PCR were as follows: (1) SIVcpzEK505: forward $5^{\prime}$-TAGTGTGTGCCCATCCATTCG- $3^{\prime}$ and reverse 5'-CACCGCCAGTCAAAATTGCG-3', (2) SIVcpzMB897 forward (5'-CCTCAGATATTAAGTGTCTGTGCGG-3') and reverse (5'-GCTAGTCAAAAATTAGGCGTACTCACC- $\left.3^{\prime}\right)$, and (3) SIVLB715 forward (5'-TGCTCGGACTCTGGTAACTA-3') and reverse (5'-CCGCTACTTCTGGTTTCACTTTCACTT$\left.3^{\prime}\right)$. All primer sets listed above were used in a qRT-PCR reaction with the following cycling conditions: $50^{\circ} \mathrm{C}$ for $10 \mathrm{~min}, 95^{\circ} \mathrm{C}$ for $5 \mathrm{~min}$, followed by 40 cycles of $95^{\circ} \mathrm{C}$ for $15 \mathrm{~s}$ and $60^{\circ} \mathrm{C}$ for $30 \mathrm{~s}$ in the Bio-Rad C1000 Thermo Cycler with the CFX96 Real-Time System (Bio-Rad, Hercules, CA, United States). The standard curve was prepared using a series of 10-fold dilutions of viral SIVcpzEK505, SIVcpzMB897, or SIVcpzLB715 LTR at a known concentration. The sensitivity of this assay was 1,000 copies per ml (1 copy per $\mu \mathrm{l})$.

\section{Determination of CD4 ${ }^{+} \mathbf{T}$ Cell Levels}

Peripheral blood was collected bi-monthly from infected and control mice by tail vein puncture. Human cell engraftment levels were assessed by flow cytometry. $5 \mu \mathrm{l}$ of Fc $\gamma \mathrm{R}$-block (Jackson ImmunoResearch Laboratories, Inc., West Grove, PA, United States) was added to the blood for $5 \mathrm{~min}$. The blood 
was then stained with fluorophore conjugated hCD45-FITC, hCD3-PE and hCD4-PE/Cy5 (BD Pharmingen, San Jose, CA, United States) for $30 \mathrm{~min}$. Erythrocytes were lysed using the Whole Blood Erythrocyte Lysing kit according to the manufacturer's instructions (R\&D Systems, Minneapolis, MN, United States). The stained cells were then fixed in $1 \%$ paraformaldehyde and $0.45 \mu \mathrm{m}$-filtered. To assess $\mathrm{CD}^{+} \mathrm{T}$ cell depletion in uninfected and infected mice, the $\mathrm{CD}^{+} \mathrm{T}$ cells levels were calculated as a ratio of the entire CD $45^{+}$(lymphocyte common antigen) population. The $\mathrm{CD} 4^{+} \mathrm{T}$ cell population levels were then determined as a percentage of the entire $\mathrm{CD} 3^{+} \mathrm{T}$ cell population. Baseline levels of the $\mathrm{CD} 45^{+}, \mathrm{CD}^{+}$and $\mathrm{CD} 4^{+}$cells were measured prior to infection as a control. All flow cytometry data was analyzed using the FlowJo v10.0.7 software package (FlowJo LLC, Ashland, OR, United States). CD4 ${ }^{+} \mathrm{T}$ cell decline was assessed utilizing a two-tailed Student's $t$-test $(p<0.001)$ to compare the two groups of mice, infected and uninfected.

\section{Illumina-Based Deep Sequencing and Analysis}

For two mice of each generation, viral RNA from the plasma collected approximately at weeks $3,11,19$, and 24 post-infection from two mice from each passage were used to synthesize the cDNA using SuperScript IV and the manufacturer's instructions (Invitrogen, Carlsbad, CA, United States). Two separate multiplexed primer pools containing overlapping regions between, but not within the pools, were designed utilizing the Primal Scheme software ${ }^{1}$ in order to generate overlapping 400 base pair amplicons that spanned our entire viral genomes as seen in Supplementary Tables 1-3 (Quick et al., 2017). The amplicons were prepped for Illumina-based deep sequencing using the Nextera XT DNA Library preparation kit and the manufacturer's instructions (Illumina, San Diego, CA, United States). The amplicon library was deep sequenced at the sequencing core facility at the University of Wisconsin, Madison, utilizing a MiSeq Illumina desktop sequencer (Invitrogen, Carlsbad, CA, United States). All sequence reads were prepared as FASTQ file format and analyzed as described below.

\section{Cell Culture}

Whole blood filter packs were obtained from the Garth Englund Blood Center of Fort Collins, CO, United States. Mononuclear cells were isolated by Ficoll-Plaque density centrifugation. PBMC were grown and maintained in RPMI media containing 10\% heat inactivated fetal bovine serum (HI FBS), $2 \times$ antibioticantimycotic mix (Thermo Fisher Scientific, Waltham, MA, United States) and $20 \mathrm{ng} / \mathrm{mL}$ IL-2 (R\&D Systems, Inc., Minneapolis, MN, United States). For viral propagation, the PBMC were CD8 depleted by positive selection and then stimulated using $100 \mathrm{ng} / \mathrm{mL}$ of anti-CD3 and anti-CD28 soluble antibody (Miltenyi Biotec Inc., Auburn, CA, United States) for 48 h. The TZM-bl reporter and 293 cell lines were cultured in DMEM media containing 10\% HI FBS, $1 \%$ antibiotic-antimycotic mix (Thermo Fisher Scientific, Waltham, MA, United States) and $1 \%$ L-glutamine.

${ }^{1}$ http://primal.zibraproject.org

\section{Viral Propagation, Titration and Subsequent Viral Passaging of SIVcpz}

To propagate the virus from the first passage to the next, bone marrow, thymus, spleen, mesenteric and axillary lymph nodes, and whole blood obtained through cardiac puncture were harvested from the SIVcpz infected mice with the highest plasma viral titer. Lymphocyte fractions isolated by Ficoll-Plaque density centrifugation were seeded at a density of $2-3 \times 10^{6}$ cells $/ \mathrm{mL}$ and activated for $48 \mathrm{~h}$ with $100 \mathrm{ng} / \mathrm{mL}$ of anti-CD3 and antiCD28 soluble antibody (Miltenyi Biotec Inc., Auburn, CA, United States). To assure viral infection of the next generation mice, these cells were then co-cultured for $48 \mathrm{~h}$ with fresh splenocytes obtained from the new hu-mice cohort used for serial passage. These cultured cells together with culture supernatants containing the virus were inoculated intraperitoneally to the next hu-mice batch of 5 mice.

\section{Quantification of Variant Frequencies}

To calculate the variant frequencies in the sequencing datasets, a strategy similar to that we previously described in Schmitt et al. (2018). Briefly, we removed the primer sequences first by trimming 30 bases off the ends of the reads, then removed the adapter sequences and low-quality bases using the cutadapt software v1.9.1 (Martin, 2011). Filtered reads were mapped to stock virus consensus sequences using the bowtie2 software v2.2.5 (Langmead and Salzberg, 2012). Bowtie2 BAM format output was used as input to lofreq software v2.1.2 to call variants (Wilm et al., 2012). To qualify for a variant, we required $>100$ coverage and $>1 \%$ frequency. The impact of variants was determined, and variants were plotted using R and ggplot2 (ISBN: 0387981403). Scripts are available at GitHub repositories ${ }^{2,3}$.

\section{Assessment of Amino Acid Frequencies in HIV-1 and SIV Database Sequences}

To quantify the frequencies of specific amino acids in SIV and HIV sequences for use as a comparison available from the HIV Sequence Database Compendium ${ }^{4}$, multiple protein sequence alignments for each $\mathrm{HIV}-1 / \mathrm{SIVcpz}$ protein were downloaded from the 2017 compendium, which were the most recent alignments available for retrieval in April of 2019 (Foley et al., 2017). From the total HIV-1/SIVcpz alignments, subsets containing only HIV-1 or SIV sequences were extracted. These were used to create a position frequency matrix by tabulating, for each position (each column) of the alignment, the number of observations of each amino acid or gap character. A mapping of the amino acid position in the SIV protein to the position (column) in the multiple sequence alignment was then established for each protein of each of the three SIVcpz strains (EK505, MB897, and LB715). The position frequency matrix plus this mapping helped determine the frequency in these sets of HIV and SIV sequences of any variant that arose during the passage/generation experiments. The scripts and data files

\footnotetext{
${ }^{2}$ https://github.com/stenglein-lab/SIV_to_HIV-1

${ }^{3}$ https://github.com/stenglein-lab/viral_variant_explorer

${ }^{4}$ http://www.hiv.lanl.gov/
} 
used to conduct this analysis are available at: https://github.com/ stenglein-lab/SIV_to_HIV-1.

\section{DATA AVAILABILITY STATEMENT}

The raw data supporting the conclusions of this article is available on the sequence read archive (SRA) (BioSample accession numbers: SAMN15338262-SAMN15338315).

\section{ETHICS STATEMENT}

The animal study was reviewed and approved by Colorado State University IACUC.

\section{AUTHOR CONTRIBUTIONS}

RA, KS, JC, MS, PM, and SO'C were responsible for the design and conduct of the project. LR-M, KG, and RM provided technical assistance. All authors contributed to the article and approved the submitted version.

\section{REFERENCES}

Akkina, R. (2013). New generation humanized mice for virus research: comparative aspects and future prospects. Virology 435, 14-28. doi: 10.1016/j.virol.2012. 10.007

Akkina, R. K., Rosenblatt, J. D., Campbell, A. G., Chen, I. S., and Zack, J. A. (1994). Modeling human lymphoid precursor cell gene therapy in the SCID-hu mouse. Blood 84, 1393-1398. doi: 10.1182/blood.v84.5.1393.1393

Aldrovandi, G. M., Feuer, G., Gao, L., Jamieson, B., Kristeva, M., Chen, I. S., et al. (1993). The SCID-hu mouse as a model for HIV-1 infection. Nature 363, 732-736. doi: 10.1038/363732a0

Baenziger, S., Tussiwand, R., Schlaepfer, E., Mazzucchelli, L., Heikenwalder, M., Kurrer, M. O., et al. (2006). Disseminated and sustained HIV infection in CD34+ cord blood cell-transplanted Rag2-/-gamma c-/- mice. Proc. Natl. Acad. Sci. U.S.A. 103, 15951-15956. doi: 10.1073/pnas.0604493103

Bai, J., Gorantla, S., Banda, N., Cagnon, L., Rossi, J., and Akkina, R. (2000). Characterization of anti-CCR5 ribozyme-transduced CD34+ hematopoietic progenitor cells in vitro and in a SCID-hu mouse model in vivo. Mol. Ther. 1, 244-254. doi: 10.1006/mthe.2000.0038

Berger, A., Sommer, A. F., Zwarg, J., Hamdorf, M., Welzel, K., Esly, N., et al. (2011). SAMHD1-deficient CD14+ cells from individuals with Aicardi-Goutieres syndrome are highly susceptible to HIV-1 infection. PLoS Pathog. 7:e1002425. doi: 10.1371/journal.ppat.1002425

Berges, B. K., Akkina, S. R., Folkvord, J. M., Connick, E., and Akkina, R. (2008). Mucosal transmission of R5 and X4 tropic HIV-1 via vaginal and rectal routes in humanized Rag2-/- gammac -/- (RAG-hu) mice. Virology 373, 342-351. doi: 10.1016/j.virol.2007.11.020

Berges, B. K., Akkina, S. R., Remling, L., and Akkina, R. (2010). Humanized Rag2(/-)gammac(-/-) (RAG-hu) mice can sustain long-term chronic HIV-1 infection lasting more than a year. Virology 397, 100-103. doi: 10.1016/j.virol.2009.10.034

Berges, B. K., Wheat, W. H., Palmer, B. E., Connick, E., and Akkina, R. (2006). HIV-1 infection and CD4 T cell depletion in the humanized Rag2-/-gamma c-/(RAG-hu) mouse model. Retrovirology 3:76. doi: 10.1186/1742-4690-3-76

Bibollet-Ruche, F., Heigele, A., Keele, B. F., Easlick, J. L., Decker, J. M., Takehisa, J., et al. (2012). Efficient SIVcpz replication in human lymphoid tissue requires viral matrix protein adaptation. J. Clin. Invest. 122, 1644-1652. doi: 10.1172/ JCI61429

Charlins, P., Schmitt, K., Remling-Mulder, L., Hogan, L. E., Hanhauser, E., Hobbs, K. S., et al. (2017). A humanized mouse-based HIV-1 viral outgrowth assay

\section{FUNDING}

Work described was supported by NIH, United States grant R01 AI123234 to RA.

\section{ACKNOWLEDGMENTS}

This project was also supported in part by the National Center for Research Resources and the Office of Research Infrastructure Programs (ORIP) of the NIH through grant OD011104 at the Tulane National Primate Research Center and the NIH grant P51OD011106 at the Wisconsin National Primate Research Center. Computational resources were supported by NIH/NCATS Colorado CTSA Grant Number UL1 TR002535.

\section{SUPPLEMENTARY MATERIAL}

The Supplementary Material for this article can be found online at: https://www.frontiersin.org/articles/10.3389/fmicb. 2020.01889/full\#supplementary-material

with higher sensitivity than in vitro qVOA in detecting latently infected cells from individuals on ART with undetectable viral loads. Virology 507, 135-139. doi: 10.1016/j.virol.2017.04.011

Chen, B., Vogan, E. M., Gong, H., Skehel, J. J., Wiley, D. C., and Harrison, S. C. (2005). Structure of an unliganded simian immunodeficiency virus gp120 core. Nature 433, 834-841. doi: 10.1038/nature03327

Chen, Z., Telfier, P., Gettie, A., Reed, P., Zhang, L., Ho, D. D., et al. (1996) Genetic characterization of new West African simian immunodeficiency virus SIVsm: geographic clustering of household-derived SIV strains with human immunodeficiency virus type 2 subtypes and genetically diverse viruses from a single feral sooty mangabey troop. J. Virol. 70, 3617-3627. doi: 10.1128/jvi.70. 6.3617-3627.1996

Choudhary, S. K., Archin, N. M., Cheema, M., Dahl, N. P., Garcia, J. V., and Margolis, D. M. (2012). Latent HIV-1 infection of resting CD4(+) T cells in the humanized Rag2(-)/(-) gammac(-)/(-) mouse. J. Virol. 86, 114-120. doi: 10.1128/JVI.05590-11

Curlin, J., Schmitt, K., Remling-Mulder, L., Moriarty, R., Stenglein, M., O'Connor, S., et al. (2020). SIVcpz cross-species transmission and viral evolution toward HIV-1 in a humanized mouse model. J. Med. Primatol. 49, 40-43. doi: 10.1111/ jmp. 12440

Decker, J. M., Bibollet-Ruche, F., Wei, X., Wang, S., Levy, D. N., Wang, W., et al. (2005). Antigenic conservation and immunogenicity of the HIV coreceptor binding site. J. Exp. Med. 201, 1407-1419. doi: 10.1084/jem.20042510

Denton, P. W., and Garcia, J. V. (2011). Humanized mouse models of HIV infection. AIDS Rev. 13, 135-148.

Derdeyn, C. A., Decker, J. M., Sfakianos, J. N., Wu, X., O’Brien, W. A., Ratner, L., et al. (2000). Sensitivity of human immunodeficiency virus type 1 to the fusion inhibitor T-20 is modulated by coreceptor specificity defined by the V3 loop of gp120. J. Virol. 74, 8358-8367. doi: 10.1128/jvi.74.18.8358-8367. 2000

Etienne, L., Bibollet-Ruche, F., Sudmant, P. H., Wu, L. I., Hahn, B. H., and Emerman, M. (2015). The role of the antiviral APOBEC3 gene family in protecting chimpanzees against lentiviruses from monkeys. PLoS Pathog. 11:e1005149. doi: 10.1371/journal.ppat.1005149

Foley, B., Thomas, L., Apetrei, C., Hahn, B., Mizrachi, I., Mullins, J., et al. (2017). HIV Sequence Compendium 2017. Los Alamos: Los Alamos National Laboratory, Theoretical and Biophysics.

Garcia, S., and Freitas, A. A. (2012). Humanized mice: current states and perspectives. Immunol. Lett. 146, 1-7. doi: 10.1016/j.imlet.2012.03.009 
Ghimire, D., Rai, M., and Gaur, R. (2018). Novel host restriction factors implicated in HIV-1 replication. J. Gen. Virol. 99, 435-446. doi: 10.1099/jgv.0.001026

Goldstone, D. C., Ennis-Adeniran, V., Hedden, J. J., Groom, H. C., Rice, G. I., Christodoulou, E., et al. (2011). HIV-1 restriction factor SAMHD1 is a deoxynucleoside triphosphate triphosphohydrolase. Nature 480, 379-382. doi: 10.1038 /nature 10623

Gonzalez, M. E. (2017). The HIV-1 Vpr protein: a multifaceted target for therapeutic intervention. Int. J. Mol. Sci. 18:126. doi: 10.3390/ijms18010126

Hahn, B. H., Shaw, G. M., De Cock, K. M., and Sharp, P. M. (2000). AIDS as a zoonosis: scientific and public health implications. Science 287, 607-614. doi: $10.1126 /$ science.287.5453.607

Hemelaar, J. (2012). The origin and diversity of the HIV-1 pandemic. Trends Mol. Med. 18, 182-192. doi: 10.1016/j.molmed.2011.12.001

Hirsch, V. M., Olmsted, R. A., Murphey-Corb, M., Purcell, R. H., and Johnson, P. R. (1989). An African primate lentivirus (SIVsm) closely related to HIV-2. Nature 339, 389-392. doi: 10.1038/339389a0

Hu, S., Neff, C. P., Kumar, D. M., Habu, Y., Akkina, S. R., Seki, T., et al. (2017). A humanized mouse model for HIV-2 infection and efficacy testing of a singlepill triple-drug combination anti-retroviral therapy. Virology 501, 115-118. doi: 10.1016/j.virol.2016.11.013

Huet, T., Cheynier, R., Meyerhans, A., Roelants, G., and Wain-Hobson, S. (1990). Genetic organization of a chimpanzee lentivirus related to HIV-1. Nature 345, 356-359. doi: 10.1038/345356a0

Hvilsom, C., Carlsen, F., Siegismund, H. R., Corbet, S., Nerrienet, E., and Fomsgaard, A. (2008). Genetic subspecies diversity of the chimpanzee CD4 virus-receptor gene. Genomics 92, 322-328. doi: 10.1016/j.ygeno.2008.07.003

Joseph, S. B., Swanstrom, R., Kashuba, A. D., and Cohen, M. S. (2015). Bottlenecks in HIV-1 transmission: insights from the study of founder viruses. Nat. Rev. Microbiol. 13, 414-425. doi: 10.1038/nrmicro3471

Kamata, M., and Aida, Y. (2000). Two putative alpha-helical domains of human immunodeficiency virus type $1 \mathrm{Vpr}$ mediate nuclear localization by at least two mechanisms. J. Virol. 74, 7179-7186. doi: 10.1128/jvi.74.15.7179-7186.2000

Kariuki, S. M., Selhorst, P., Arien, K. K., and Dorfman, J. R. (2017). The HIV-1 transmission bottleneck. Retrovirology 14:22. doi: 10.1186/s12977-017-0343-8

Keele, B. F., Van Heuverswyn, F., Li, Y., Bailes, E., Takehisa, J., Santiago, M. L., et al. (2006). Chimpanzee reservoirs of pandemic and nonpandemic HIV-1. Science 313, 523-526. doi: 10.1126/science.1126531

Kino, T., Gragerov, A., Kopp, J. B., Stauber, R. H., Pavlakis, G. N., and Chrousos, G. P. (1999). The HIV-1 virion-associated protein vpr is a coactivator of the human glucocorticoid receptor. J. Exp. Med. 189, 51-62. doi: 10.1084/jem.189. 1.51

Kluge, S. F., Sauter, D., Vogl, M., Peeters, M., Li, Y., Bibollet-Ruche, F., et al. (2013). The transmembrane domain of HIV-1 Vpu is sufficient to confer anti-tetherin activity to SIVcpz and SIVgor Vpu proteins: cytoplasmic determinants of Vpu function. Retrovirology 10:32. doi: 10.1186/1742-4690-10-32

Kogan, M., and Rappaport, J. (2011). HIV-1 accessory protein Vpr: relevance in the pathogenesis of HIV and potential for therapeutic intervention. Retrovirology 8:25. doi: 10.1186/1742-4690-8-25

Langmead, B., and Salzberg, S. L. (2012). Fast gapped-read alignment with Bowtie 2. Nat. Methods 9, 357-359. doi: 10.1038/nmeth.1923

Le Tortorec, A., and Neil, S. J. (2009). Antagonism to and intracellular sequestration of human tetherin by the human immunodeficiency virus type 2 envelope glycoprotein. J. Virol. 83, 11966-11978. doi: 10.1128/JVI.01515-09

Lim, E. S., Malik, H. S., and Emerman, M. (2010). Ancient adaptive evolution of tetherin shaped the functions of $\mathrm{Vpu}$ and Nef in human immunodeficiency virus and primate lentiviruses. J. Virol. 84, 7124-7134. doi: 10.1128/JVI. 00468-10

Locatelli, S., and Peeters, M. (2012). Cross-species transmission of simian retroviruses: how and why they could lead to the emergence of new diseases in the human population. AIDS 26, 659-673. doi: 10.1097/QAD. 0b013e328350fb68

Martin, M. (2011). Cutadapt removes adapter sequences from high-throughput sequencing reads. EMBnet J. 17, 10-12.

Marx, P. A., Alcabes, P. G., and Drucker, E. (2001). Serial human passage of simian immunodeficiency virus by unsterile injections and the emergence of epidemic human immunodeficiency virus in Africa. Philos. Trans. R. Soc. Lond. B Biol. Sci. 356, 911-920. doi: 10.1098/rstb.2001. 0867
McNatt, M. W., Zang, T., and Bieniasz, P. D. (2013). Vpu binds directly to tetherin and displaces it from nascent virions. PLoS Pathog. 9:e1003299. doi: 10.1371/ journal.ppat.1003299

Meyerson, N. R., Warren, C. J., Vieira, D., Diaz-Griferro, F., and Sawyer, S. L. (2018). Species-specific vulnerability of RanBP2 shaped the evolution of SIV as it transmitted in African apes. PLoS Pathog. 14:e1006906. doi: 10.1371/journal. ppat. 1006906

Nagata, S., Imai, J., Makino, G., Tomita, M., and Kanai, A. (2017). Evolutionary analysis of HIV-1 pol proteins reveals representative residues for viral subtype differentiation. Front. Microbiol. 8:2151. doi: 10.3389/fmicb.2017.02151

Neff, C. P., Ndolo, T., Tandon, A., Habu, Y., and Akkina, R. (2010). Oral preexposure prophylaxis by anti-retrovirals raltegravir and maraviroc protects against HIV-1 vaginal transmission in a humanized mouse model. PLoS One 5:e15257. doi: 10.1371/journal.pone.0015257

Neil, S. J., Zang, T., and Bieniasz, P. D. (2008). Tetherin inhibits retrovirus release and is antagonized by HIV-1 Vpu. Nature 451, 425-430. doi: 10.1038/ nature 06553

Quick, J., Grubaugh, N. D., Pullan, S. T., Claro, I. M., Smith, A. D., Gangavarapu, K., et al. (2017). Multiplex PCR method for MinION and Illumina sequencing of Zika and other virus genomes directly from clinical samples. Nat. Protoc. 12, 1261-1276. doi: 10.1038/nprot.2017.066

Rosa, A., Chande, A., Ziglio, S., De Sanctis, V., Bertorelli, R., Goh, S. L., et al. (2015). HIV-1 Nef promotes infection by excluding SERINC5 from virion incorporation. Nature 526, 212-217. doi: 10.1038/nature15399

Sato, K., Misawa, N., Takeuchi, J. S., Kobayashi, T., Izumi, T., Aso, H., et al. (2018). Experimental adaptive evolution of simian immunodeficiency virus SIVcpz to pandemic human immunodeficiency virus type 1 by using a humanized mouse model. J. Virol. 92, e1905-e1917. doi: 10.1128/JVI.01905-17

Sauter, D., and Kirchhoff, F. (2019). Key viral adaptations preceding the AIDS pandemic. Cell Host Microbe 25, 27-38. doi: 10.1016/j.chom.2018.12.002

Sayah, D. M., Sokolskaja, E., Berthoux, L., and Luban, J. (2004). Cyclophilin A retrotransposition into TRIM5 explains owl monkey resistance to HIV-1. Nature 430, 569-573. doi: 10.1038/nature02777

Schmitt, K., Curlin, J., Kumar, D. M., Remling-Mulder, L., Feely, S., Stenglein, M., et al. (2018). SIV progenitor evolution toward HIV: a humanized mouse surrogate model for SIVsm adaptation toward HIV-2. J. Med. Primatol. 47, 298-301. doi: 10.1111/jmp.12380

Schmitt, K., Mohan Kumar, D., Curlin, J., Remling-Mulder, L., Stenglein, M., O'Connor, S., et al. (2017). Modeling the evolution of SIV sooty mangabey progenitor virus towards HIV-2 using humanized mice. Virology 510, 175-184. doi: 10.1016/j.virol.2017.07.005

Serra-Moreno, R., and Evans, D. T. (2012). Adaptation of human and simian immunodeficiency viruses for resistance to tetherin/BST-2. Curr. HIV Res. 10, 277-282. doi: 10.2174/157016212800792496

Sharp, P. M., and Hahn, B. H. (2011). Origins of HIV and the AIDS pandemic. Cold Spring Harb. Perspect. Med. 1:a006841. doi: 10.1101/cshperspect.a006841

Sharp, P. M., Robertson, D. L., Gao, F., and Hahn, B. H. (1994). Origins and diversity of human immunodeficiency viruses. AIDS 8(Suppl. 1), S27-S42.

Sheehy, A. M., Gaddis, N. C., Choi, J. D., and Malim, M. H. (2002). Isolation of a human gene that inhibits HIV-1 infection and is suppressed by the viral Vif protein. Nature 418, 646-650. doi: 10.1038/nature00939

Sherman, M. P., de Noronha, C. M., Pearce, D., and Greene, W. C. (2000). Human immunodeficiency virus type $1 \mathrm{Vpr}$ contains two leucine-rich helices that mediate glucocorticoid receptor coactivation independently of its effects on $\mathrm{G}(2)$ cell cycle arrest. J. Virol. 74, 8159-8165. doi: 10.1128/jvi.74.17.81598165.2000

Shultz, L. D., Brehm, M. A., Garcia-Martinez, J. V., and Greiner, D. L. (2012). Humanized mice for immune system investigation: progress, promise and challenges. Nat. Rev. Immunol. 12, 786-798. doi: 10.1038/nri3311

Steckbeck, J. D., Craigo, J. K., Barnes, C. O., and Montelaro, R. C. (2011). Highly conserved structural properties of the C-terminal tail of HIV-1 gp41 protein despite substantial sequence variation among diverse clades: implications for functions in viral replication. J. Biol. Chem. 286, 27156-27166. doi: 10.1074/jbc. M111.258855

Stremlau, M., Owens, C. M., Perron, M. J., Kiessling, M., Autissier, P., and Sodroski, J. (2004). The cytoplasmic body component TRIM5alpha restricts HIV-1 infection in Old World monkeys. Nature 427, 848-853. doi: 10.1038/ nature 02343 
Usami, Y., Wu, Y., and Gottlinger, H. G. (2015). SERINC3 and SERINC5 restrict HIV-1 infectivity and are counteracted by Nef. Nature 526, 218-223. doi: 10. 1038/nature15400

Van Heuverswyn, F., Li, Y., Neel, C., Bailes, E., Keele, B. F., Liu, W., et al. (2006). Human immunodeficiency viruses: SIV infection in wild gorillas. Nature 444:164. doi: 10.1038/444164a

Veselinovic, M., Charlins, P., and Akkina, R. (2016). Modeling HIV-1 mucosal transmission and prevention in humanized mice. Methods Mol. Biol. 1354, 203-220. doi: 10.1007/978-1-4939-3046-3_14

Vigan, R., and Neil, S. J. (2010). Determinants of tetherin antagonism in the transmembrane domain of the human immunodeficiency virus type $1 \mathrm{Vpu}$ protein. J. Virol. 84, 12958-12970. doi: 10.1128/JVI. 01699-10

Wain, L. V., Bailes, E., Bibollet-Ruche, F., Decker, J. M., Keele, B. F., Van Heuverswyn, F., et al. (2007). Adaptation of HIV-1 to its human host. Mol. Biol. Evol. 24, 1853-1860. doi: 10.1093/molbev/msm110

Wei, X., Decker, J. M., Liu, H., Zhang, Z., Arani, R. B., Kilby, J. M., et al. (2002). Emergence of resistant human immunodeficiency virus type 1 in patients receiving fusion inhibitor (T-20) monotherapy. Antimicrob. Agents Chemother. 46, 1896-1905. doi: 10.1128/aac.46.6.1896-1905.2002

Wilm, A., Aw, P. P., Bertrand, D., Yeo, G. H., Ong, S. H., Wong, C. H., et al. (2012). LoFreq: a sequence-quality aware, ultra-sensitive variant caller for uncovering cell-population heterogeneity from high-throughput sequencing datasets. Nucleic Acids Res. 40, 11189-11201. doi: 10.1093/nar/gks918

Worobey, M., Telfer, P., Souquiere, S., Hunter, M., Coleman, C. A., Metzger, M. J., et al. (2010). Island biogeography reveals the deep history of SIV. Science 329:1487. doi: 10.1126/science.1193550
Xiang, S. H., Doka, N., Choudhary, R. K., Sodroski, J., and Robinson, J. E. (2002) Characterization of CD4-induced epitopes on the HIV type 1 gp120 envelope glycoprotein recognized by neutralizing human monoclonal antibodies. AIDS Res. Hum. Retroviruses 18, 1207-1217. doi: 10.1089/08892220260387959

Yu, B., Fonseca, D. P., O’Rourke, S. M., and Berman, P. W. (2010). Protease cleavage sites in HIV-1 gp120 recognized by antigen processing enzymes are conserved and located at receptor binding sites. J. Virol. 84, 1513-1526. doi: 10.1128/JVI.01765-09

Yuan, Z., Kang, G., Daharsh, L., Fan, W., and Li, Q. (2018). SIVcpz closely related to the ancestral HIV-1 is less or non-pathogenic to humans in a hu-BLT mouse model. Emerg. Microbes Infect. 7:59. doi: 10.1038/s41426-018-0062-9

Yuan, Z., Kang, G., Ma, F., Lu, W., Fan, W., Fennessey, C. M., et al. (2016). Recapitulating cross-species transmission of simian immunodeficiency virus SIVcpz to humans by using humanized BLT mice. J. Virol. 90, 7728-7739. doi: 10.1128/JVI.00860-16

Conflict of Interest: The authors declare that the research was conducted in the absence of any commercial or financial relationships that could be construed as a potential conflict of interest.

Copyright $\odot 2020$ Schmitt, Curlin, Remling-Mulder, Moriarty, Goff, O'Connor, Stenglein, Marx and Akkina. This is an open-access article distributed under the terms of the Creative Commons Attribution License (CC BY). The use, distribution or reproduction in other forums is permitted, provided the original author(s) and the copyright owner(s) are credited and that the original publication in this journal is cited, in accordance with accepted academic practice. No use, distribution or reproduction is permitted which does not comply with these terms. 